\title{
Complexity Analysis of the Overall Behavior of the Network based on the Topology Structure \\ Ying Zhou
}

Linyi University, Shandong 273400, China

13007641@qq.com

Keywords: Topology; Complex network.

\begin{abstract}
One of the main reasons behind complex networks popularity is their flexibility and generality for representing virtually any natural structure. Each complex network presents specific topological features which characterize its connectivity and highly influence the dynamics of processes executed on the network. Using topological structure metrics to mining sub graph patterns of different structure types. The relationship between the topology of complex networks and the control ability of dynamic structures has become a hot research topic. Centrality of topology structure is one of the core content of complex network analysis. In particular, consider their relations between nodes direction specificity of connection and system dynamic control function of the exercise, a measure of a node from point of view of the control structure to intervene the size of network is very practical value.
\end{abstract}

\section{The Background and Significance}

The complex network analysis of the real system structure and the dynamic evolution of system structure of the potential flexibly and naturally. Network structure analysis involves the aspects of the work include: it represented interest system to the network, and to study the topological structure of the network by quantitative index; quantitative evolution in the process of network topology changes, to analyze the evolution of impact on the network connection; application metrics to discover the different structure types of sub-graph patterns. It is similar with the pattern recognition work. In recent years, complex network has become a hot topic in the research of cross discipline. Including the fields such as physics, mathematics, biology, economics, social science and information science, many scientists from different angles on complex network characteristics were analyzed. There are many researches on complex network. It is shown in figure 1.

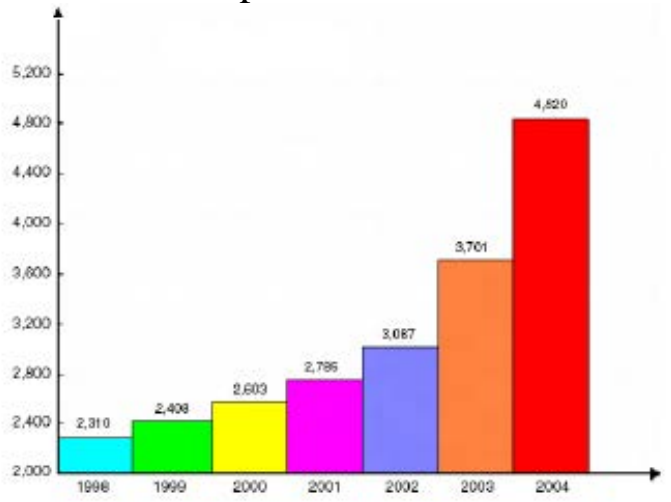

Figure 1 Statistics on the growth situation of the complex network papers

\section{Introduction}

\subsection{The complex network}

Study on the network and map the earliest origins in 1735 the German mathematician Euler solving the problem of the Seven Bridges of Königsberg Bridge. Subsequently, the network research has gradually developed into a systematic scientific theory. By the end of the 1950s, two Hungarian scientists Erdős and Rényi have proposed ER model. It is shown in figure 2. In the ER 
model, the link between the two nodes is generated according to a probability. With the ER model proposed, it has made great progress in the modeling of the real network and the research of the network theory. However to the 90s of last century, with the development of computer processing ability and calculation data on an unprecedented scale increases, it was found the traditional network model in dealing with large-scale network appeared a lot of problems. These problems make people to study complex network requires more sophisticated statistical tools to describe the characteristics of its own, and also need new theoretical model to describe the complex network.

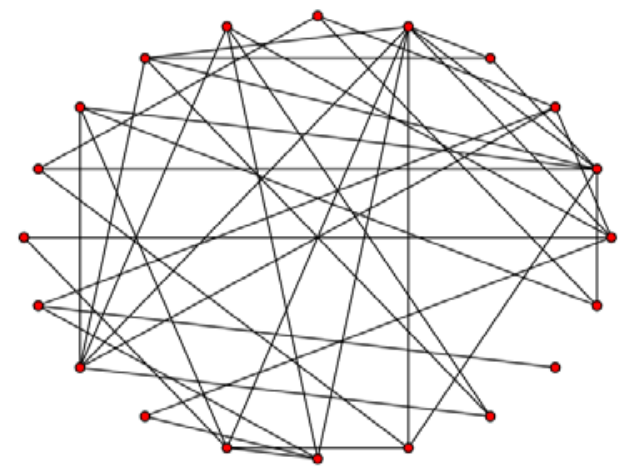

Figure 2 ER model

\subsection{Topology Structure}

Centrality, similarity and robustness have important roles in network analysis.

The value of the node in the network depends on the location of the node in the network, node location is the center, the greater the value analysis. The centrality of topology structure is used to find the most important nodes in the network. Topological structure similarity is used to describe the same or similar functions and capabilities that may exist between nodes. It is one of the most important research works in the field of network analysis, and many typical applications are based on the similarity measure. For a given complex network to remove a node, it also removes all edges adjacent to the node. It will make some interruption of the path of the original network, or directly lead to some nodes not connected. If some nodes are removed, the network can maintain most of the nodes connected, so it is considered that the topology of the network is robust to node failures.

\section{Complexity Analysis of the Overall Behavior of the Network}

The study of complex networks is more based on the analysis of the macro and micro topology structure and the relevant rules to get a systematic understanding of the complex network.

\subsection{Distribution of connectivity and free scale network}

The distribution of node connectivity in complex networks is an important geometric property of the macro topology. Under normal circumstances, we define the connection degree distribution $p_{k}$. $p_{k}$ is the probability of node degree $\mathrm{k}$ in the network. $\left\{p_{k}\right\}(\mathrm{k}=0,1, \ldots)$ formed degree distribution of the nodes.

The connection degree distribution of many real networks is not consistent with the Poisson distribution predicted by the ER model. The actual situation is that a large number of real networks exist in the form of power function of degree distribution. It as follow:

$$
\mathrm{P}_{\mathrm{k}} \sim \mathrm{k}^{-\alpha} \text {, ( } \alpha \text { is constant) }
$$

$\mathrm{k}$ is the node connectivity, and the constant $\alpha$ reflects the exponential law of the degree distribution. In such networks, the distribution of connectivity is determined by the constant $\alpha$, which is independent of the scale of the network. So it is called the Scale Free Network.

\subsection{Shortest path and Small world effect}

In a graph, the shortest path $l_{i j}$ between any two $i$ and $j$ for all paths connected between the two nodes and the other vertex of at least one or several paths. Note $(i, j)$ the shortest path between the collection is $S_{i j}$. The corresponding path length is $d_{i j}\left|I_{i j}\right|$. When the two nodes are not connected, the shortest path between the nodes is either infinite, or a value is set by a predetermined set of rules. Average shortest path $l$ of a graph is 


$$
l=\frac{1}{\frac{1}{2} n(n+1)} \sum_{i \neq j} d_{i j}
$$

The small world phenomenon in complex networks between the network nodes, the average shortest distance is very short, in the connectivity of, a medium scale of each node of the network only takes up steps can reach any other node.

\subsection{Transitivity and Clustering Coefficient}

In the theory of classical network research, the transitivity of the network refers to the distribution density of the closed loop topology in the whole network. Under normal circumstances, the basic closed loop, triangular structure, the distribution of density in the network can basically reflect the overall transmission of the network. The important means to analyze the network transmission is the clustering coefficient of the network.

The clustering coefficient mainly reflects the density of the triangular structure in the network. Cluster coefficient can be measured for a single node. It can also be measured from the network as a whole. On the specific definition and measurement method of cluster coefficient as follow:

$$
C=\frac{3 \times \text { number of triangles in the network }}{\text { number of connected triples of vertices }}
$$

Complex network in the obvious the poly group coefficients show that in large sparse real network, the micro topology has very obvious small group agglomeration. The phenomenon of small group agglomeration plays a key role in the hierarchical structure of the network topology, the connectivity of the network and the division of the network community.

\subsection{Motif (Sub-graph)}

Sheen-Orr and Milo statistical analyzed the microstructure of the complex network in 2002 . It is found that there are a number of repeated small clusters in the network. Compared with the same size of the random network, they are much more than the latter in the network. And further observations, carried by the high frequency of small sub group distribution and network topology structure and network function has certain mapping relation. The researchers say the boy group Motif. Vázquez further comprehensive analysis found presence of a large number of features in a number of cellular networks. They find that there is a certain mapping between these characteristics and the global topological structure of the network. They find that there is a certain mapping between these characteristics and the global topological structure of the network.

\section{Conclusion}

On the network topology analysis and research on network modeling is mainly concerned with the understanding of the real world, and its ultimate aim is to through the research promotion based on the network application ability, and to predict and control the behavior of network system. Computer network topology is cited in research with topology size, shape independent point, line method, the abstract for a point in the network of computers and communications equipment, the transmission medium is abstracted as a line, composed of points and lines of the geometry is computer network topology. The topology of the network reflects the relationship of the structure of the entities in the network. It is the first step in the construction of computer network and the basis for the realization of a variety of network protocol. It effects on performance on the network, the reliability of the system and communication costs.

\section{References:}

[1] Erdős, P. and Rényi, A.On random graphs[J]. Publicationes Mathematicae 1959， 290-297.

[2] Sen, P., Dasgupta, S. Chatterjee, A., Sreeram, P. A. Mukherjee, G., and Manna, S.S. Small-world properties of the Indian railway network, Preprint cond-mat/0208535(2002). 
[3] Menczer. Filippo. Evolution of document networks[J]. PNAS 101,5261-5265(2004).

[4] R Herges. Topology in chemistry: designing Möbius molecules[J]. Chemical Reviews 2015, 106(12):4820-42.

[5] JCC Mckinsey. A Solution of the Decision Problem for the Lewis systems S2 and S4,with an Application to Topology[J]. Journal of Symbolic Logic, 2014, 6(4):117-124.

[6] P Talebifard, VCM Leung. Expansion Properties of Topology for Networking of Information in Cloud[J]. IEEE Transactions on Parallel \& Distributed Systems, 2014, 25(11):2877-2887. 